\section{EJHP gains MEDLINE listing}

\author{
Philip Wiffen
}

I am delighted to announce to readers of the journal that EJHP has been approved by the National Library of Medicine (NLM) for MEDLINE listing. This is a significant milestone and I want to acknowledge the contribution of three groups for this attainment.

First, the authors have responded to challenges to produce high-quality research such that we have a rich source of material to consider for publication. I continue to be impressed by the high quality and methodological rigour of many submissions. Second, I want to praise the BMJ team who have worked tirelessly behind the scenes over the last few years to achieve this. Third, my thanks go to the editorial team, whose breadth of experience and expertise ensures that the content of each issue is of high quality and where possible has something of relevance to all hospital pharmacists.

The NLM review committee highlighted the diverse composition of the editorial team and the wide international author base of the papers published in the journal. These are both things that I have strived to improve during my editorship, particularly by offering a mentoring service to new authors to encourage submissions from countries where publishing is perhaps not part of the culture for hospital pharmacists. Alongside these positive comments, the committee mentioned some other areas for improvement in our published

Pain Research Unit, Churchill Hospital, Oxford, UK

Correspondence to Professor Philip Wiffen, Pain Research Unit, Churchill Hospital, Oxford OX3 7LE, UK; pwiffen@oxfordsrs.org.uk content and we will be striving to work on these as the journal continues to develop.

This news will be welcomed by many authors based in institutions who insist that every publication must be in a MEDLINE listed journal. It is likely that we will see an increase in high-quality submissions to the journal and, therefore, it is worth reiterating the most common reasons why some papers are rejected. I continue to see poor-quality abstracts. As well as a succinct and accurate summary of the paper, the abstract needs to be enticing to encourage a reader to continue on to read the full text; they should not be treated as an afterthought! For tips on how to write a good abstract, please see my earlier editorial on this subject. ${ }^{1}$ Papers often need considerable editing for language; if you don't feel competent in that area then please seek help, either from colleagues locally or using one of the professional manuscript editing services available to authors. Don't forget to run a spell check before submitting! Poor language often leads to a speedy rejection as editors and reviewers will not be able to understand the key points of the manuscript and evaluate the scientific content.

Please also consider the wider implications of your study. For example, a study of prescribing errors in a small district hospital based in an obscure location is difficult to assess unless the results are put into a wider context. Even though the authors may have produced a good study, it needs to be relevant to the journal's audience.

The methods section is as important as the results, so ensure that your description of methods contains enough information to enable another researcher to repeat your study. The number of participants in the study should be sufficient to produce a meaningful and reliable result; if not, it is worth expanding the study even if it takes longer.

Systematic reviews are welcomed but must follow the Preferred Reporting Items for Systematic Reviews and MetaAnalyses ${ }^{2}$; this means following the whole guidance and not just submitting a flow chart.

Studies on topics that have been covered extensively before and that do not add significantly to the body of knowledge on that topic will struggle to gain acceptance. Hopefully these points will help authors to achieve a successful publication. I look forward to seeing more high-quality papers from different countries, and would encourage you all to also consider registering to be a reviewer for EJHP so that you can help shape the content of the journal as it moves forward.

\section{Competing interests None declared.}

Provenance and peer review Commissioned; internally peer reviewed.

(c) European Association of Hospital Pharmacists 2020. No commercial re-use. See rights and permissions. Published by BMJ.

(D) Check for updates

To cite Wiffen P. Eur J Hosp Pharm 2020;27:190.

Eur J Hosp Pharm 2020;27:190.

doi:10.1136/ejhpharm-2020-002391

ORCID iD

Philip Wiffen http://orcid.org/0000-0001-6085-1307

\section{REFERENCES}

1 Wiffen P. If only Abstracts were more concrete! Eur J Hosp Pharm 2016;23:125.

2 Preferred reporting items for systematic reviews and meta-analyses (PRISMA). Available: http://prismastatement.org [Accessed May 2020]. 